\title{
Prevention of Hypertrophic Scars and Keloids by the Prophylactic Use of Topical Silicone Gel Sheets Following a Surgical Procedure in an Office Setting
}

\author{
Michael H. Gold, MD, Teresa D. Foster, RN, Melissa A. Adair, RN, Kim Burlison, RN, \\ AND TAmmy LeWIS, LPN \\ Gold Skin Care Center, Nashville, Tennessee
}

BACKGROUND. Topical silicone gel sheeting has been used for more than 20 years to help reduce the size of hypertrophic scars and keloids. Its clinical efficacy and safety is well established. овjестіve. To determine whether topical silicone gel sheeting can be used to prevent hypertrophic scars and keloids from forming following dermatologic skin surgery.

METHODS. Patients undergoing skin surgery were stratified into two groups: those with no history of abnormal scarring (lowrisk group) and those with a history of abnormal scarring (high-risk group). Following the procedure, patients within each group were randomized to receive either routine postoperative care or topical silicone gel sheeting (48 hours after surgery). Patients were followed for 6 months.
RESULTS. In the low-risk group, there were no statistical differences between individuals using routine postoperative care or using topical silicone gel sheets. In the high-risk group, there was a statistical difference (39\% versus $71 \%$ ) between patients who did not develop abnormal scars and used topical silicone gel sheeting and patients who developed abnormal scars after routine postoperative treatment. Those individuals having a scar revision procedure also showed a statistical difference if topical silicone gel sheeting was used following surgery.

CONCLUSION. Topical silicone gel sheeting, with a 20 -year history of satisfaction in dermatology, now appears to be useful in the prevention of hypertrophic scars and keloids in patients undergoing scar revision.
TOPICAL PURE SILICONE gel sheets have been used in the treatment of hypertrophic scars and keloids since 1982. ${ }^{1}$ Numerous reports from the dermatology, plastic surgery, and burn literature have confirmed its efficacy in these often difficult to treat lesions. ${ }^{1-14}$ Various theories have been proposed over the years as to the mechanism of action for how topical pure silicone gel sheets act to reduce hypertrophic scars and keloids. Early investigations revealed that silicone gel sheets had no effect with regard to pressure, change in scar temperature, or oxygen tension within these scars. ${ }^{2,3}$ A decreased evaporative water loss, up to one-half that of normal skin, was seen, with the stratum corneum providing the reservoir for the fluid. The silicone gel sheet is impermeable to water and has been described as acting like the stratum corneum in reducing hemostasis, decreasing any of the associated hyperemia and fibrosis, and thus leading to an alteration, that is, flattening, of the raised scar. ${ }^{4}$ Therefore increased hydration and occlusion of the lesion being treated are the mechanisms by which most researchers feel this material works.

M.H. Gold, MD, T.D. Foster, RN, M.A. Adair, RN, K. Burlison, RN, and T. Lewis, LPN have indicated no significant interest with commercial supporters. This research was supported by Smith \& Nephew, Largo, FL. Address correspondence and reprint requests to: Michael $\mathrm{H}$. Gold, MD, Gold Skin Care Center, 2000 Richard Jones Rd., Suite 220, Nashville, TN 37215.
In 1994 it was reported that topical silicone gel sheets were useful in preventing recurrences of keloids following the surgical removal of these lesions with $\mathrm{CO}_{2}$ laser. ${ }^{9}$ In the eight subjects studied, two keloids were managed with the $\mathrm{CO}_{2}$ laser if the keloids were in the same anatomic site. Twenty-four hours postoperatively, one of the keloids had the topical silicone gel sheeting applied and one had routine skin care of antibiotic ointments and bandage. Results from these individuals showed that $12.5 \%$ of the keloids managed with the topical silicone gel sheets recurred, whereas $37.5 \%$ of the keloids that were not managed with topical gel sheets recurred in the observation period. This was the initial evidence that perhaps topical silicone gel sheets could be used prophylactically in the prevention of hypertrophic scars and keloids.

To further study the potential prophylactic use of topical pure silicone gel sheets in preventing hypertrophic scars and keloids from forming following a surgical procedure, a controlled, randomized, prospective, single-center trial was undertaken.

\section{Methods and Materials}

This study was designed to be a single-center, controlled, randomized clinical trial where patients were to assess the use of topical pure silicone gel sheets in preventing hypertrophic scars and keloids from forming following surgical procedures 
performed in a dermatologic surgery office by a dermatologic surgeon. To control this trial, patients were stratified into two groups. The low-risk group consisted of individuals who had no previous history of abnormal scarring, that is, people who have had injuries or surgical procedures in the past and had no history or evidence of hypertrophic scar or keloid formation postsurgery. The second group of patients, the high-risk group, had a significant history of abnormal scarring, that is, a positive history of hypertrophic scar or keloid formation following a previous surgical procedure.

The study design for this trial called for the stratification of patients into either low-risk or high-risk groups prior to the surgical procedure in which sutures or staples would be placed. Within either of these risk groups the patients were randomized to receive either silicone gel sheet postoperatively or routine postoperative care. The patients reviewed and signed informed consents prior to the beginning of this research trial explaining the potential risks and benefits of the protocol as well as therapeutic alternatives. All studyrelated documents were approved by the Columbia Nashville Division Institutional Review Board (Nashville, TN).

Where the patient was randomized to the routine postoperative care arm of the trial, the surgical sutured or stapled wound was treated immediately postoperatively with a standard antibacterial ointment, polymyxin B sulfate-bacitracin zinc, and a covering tape dressing. The patients were instructed to keep the sutured areas dry for 24 hours following the surgical procedure. Sutures/staples remained intact for 7-14 days, depending on the area of the body being treated and lesion being removed. The patients were followed for up to 6 months after their procedure.

Where the patient was randomized to the topical silicone gel sheet arm of the trial, the patients were once again instructed to keep the sutures dry for 24 hours postoperatively. The area had an antibiotic ointment (polymyxin B sulfate-bacitracin zinc) and bandage dressing applied for the first 48 hours postoperatively. Then at 48 hours the patient applied the topical silicone gel sheet directly over the sutured wound. The proper application of the topical silicone gel sheet has been described previously. ${ }^{13}$ The topical pure adhesive silicone gel sheet used was Cica-Care (Smith \& Nephew, Hull, England).

Table 1. Location of the Scars/Keloids in the Treatment Group

\begin{tabular}{lr}
\hline Back & $27 \%$ \\
Arms & $14 \%$ \\
Breast/chest & $12 \%$ \\
Face & $11 \%$ \\
Legs & $9 \%$ \\
Abdomen & $9 \%$ \\
Neck & $6 \%$ \\
Thorax & $5 \%$ \\
Hands & $3 \%$ \\
Foot & $2 \%$ \\
Head & $2 \%$ \\
Ear & $2 \%$ \\
\hline
\end{tabular}

Table 2. High-Risk Group Results

\begin{tabular}{lcc}
\hline & $\begin{array}{c}\text { Topical silicone } \\
\text { gel sheet }\end{array}$ & $\begin{array}{c}\text { Routine post- } \\
\text { operative care }\end{array}$ \\
\hline No abnormal scar (successful) & $71 \%$ & $39 \%$ \\
Hypertrophic scar & $29 \%$ & $44 \%$ \\
Keloid & $0 \%$ & $17 \%$ \\
\hline
\end{tabular}

The topical silicone gel sheet was to be worn for a minimum of 12 hours/day, up to 24 hours/day, if tolerated. The sheet was to be cleansed daily with a mild soapless cleanser. Each piece of topical silicone gel sheet comes from a large $12 \mathrm{~cm} \times 15 \mathrm{~cm}$ sheet of topical silicone gel. Each piece is cut to fit the area of the scar and immediate surrounding areas. The patients in this group had their sutures removed at the appropriate 7-14 days but continued to wear the topical silicone gel sheet for 6 months following the initial procedure.

All patients were followed at 2, 4, 8, 12, 16, 20, and 24 weeks after the removal of the sutures. Assessments were made by physician observations of the site, patient opinions, and scaled photographic analysis. The patient's opinion of the site was assessed in terms of discomfort, embarrassment, color, height, texture, and function and was recorded on a four-point scale.

\section{Results}

The plan was to enroll 100 patients, 50 patients into each of the two groups. Ninety-six patients entered the study during the enrollment period, 50 into the low-risk group and 46 into the high-risk group.

The low-risk patient population included $60 \%$ men and $40 \%$ women, with a mean age of 38.5 years. The high-risk patient population included $20 \%$ men and $80 \%$ women, with a mean age of 34.8 years. Overall the patient population included $41 \%$ mean and $59 \%$ women, with a mean patient age of 36.7 years. Various types of skin lesions were removed from these patients, including benign and malignant tumors, sebaceous cysts, and existing hypertrophic or keloid scars.

During the course of the study, 19 patients from the low-risk group and 11 from the high-risk group were lost to follow-up or withdrew before the 2-month inspection could be made. That left 66 patients, or $69 \%$ of the original group, available for analysis. This was 31 patients in the low-risk group and 35 patients in the high-risk group. Table 1 shows the location of the scars/keloids in the treatment group.

Table 3. Scar Revision Results

\begin{tabular}{lcc}
\hline & $\begin{array}{c}\text { Topical silicone } \\
\text { gel sheet }\end{array}$ & $\begin{array}{c}\text { Routine post- } \\
\text { operative care }\end{array}$ \\
\hline No abnormal scar & $64 \%$ & $17 \%$ \\
Hypertrophic scar/keloid & $36 \%$ & $83 \%$ \\
\hline
\end{tabular}




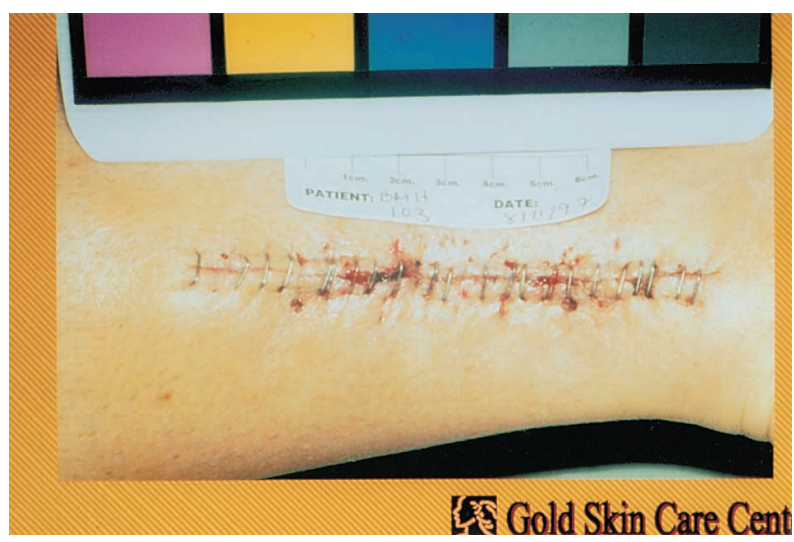

Figure 1. High-risk patient immediately post-surgery.

Within the low-risk group, one patient receiving routine wound care follow-up developed a hypertrophic scar. None of the patients using the topical silicone gel sheets developed a hypertrophic scar or keloid in the follow-up period. No statistical differences were inferred from these groups of patients.

In the high-risk group, 35 patients (17 with topical silicone gel sheets and 18 with routine postoperative care) achieved at least one follow-up inspection. In the topical silicone gel sheet arm of the trial, a $71 \%$ success rate (12 patients) was seen in preventing hypertrophic scar or keloid formation following a surgical procedure, while $29 \%$ (5 patients) developed a hypertrophic scar and none developed keloid scars. In the routine postoperative care group, a 39\% success rate (7 patients) was seen in preventing hypertrophic scars or keloids, while $17 \%$ (3 patients) developed keloid scars and $44 \%$ ( 8 patients) developed hypertrophic scars. Therefore $61 \%$ (11 patients) of the routine postoperative care group developed abnormal scars during the follow-up period. Statistical analysis provided marginal evidence $(P=.072)$ that the proportion of

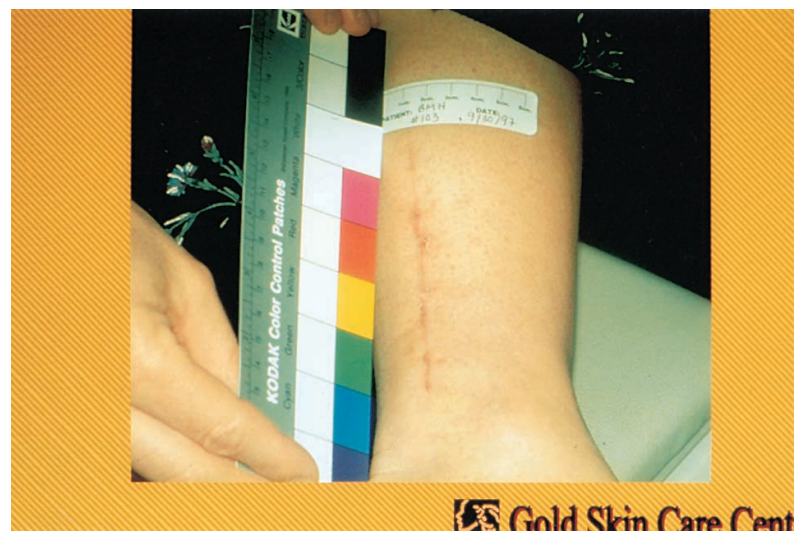

Figure 2. High-risk patient 6 months post-surgery with topical gel sheets.

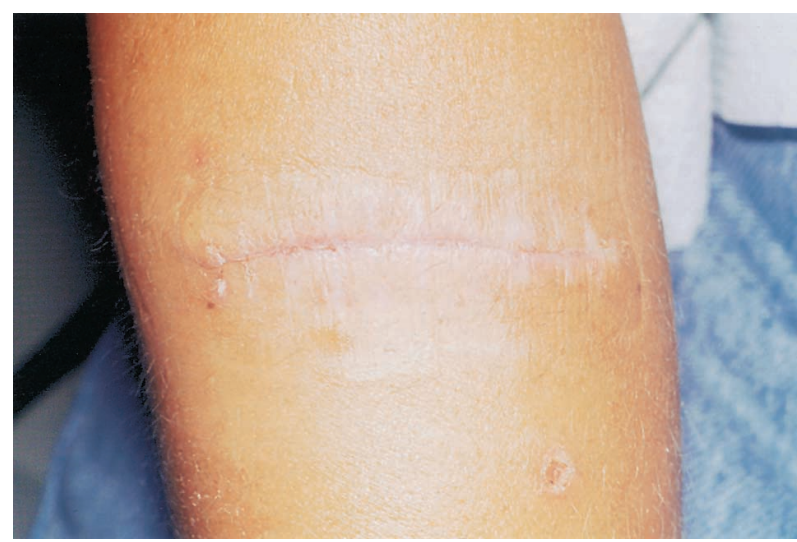

Figure 3. High-risk patient 10 days post-operative (sutures removed).

patients developing abnormal scars was lower in the topical silicone gel sheeting arm of the trial. The results are summarized in Table 2.

Within the high-risk group, 27 individuals underwent scar revisions and 19 had some other lesion treated surgically. Results were analyzed in this group to see if there were differences in the postoperative course for these difficult to treat lesions. In this group it was observed that for those patients having a scar revision procedure, $36 \%$ (4 patients) who received topical silicone gel sheets developed a recurrent abnormal scar versus $83 \%$ (10 patients) within the routine wound care group. This showed a statistical difference of $P=.035$. The results of the analysis indicate that topical silicone gel sheets are effective in reducing the occurrence of abnormal scars in patients who had a preexisting scar surgically treated. These results are summarized in Table 3. There was no evidence of a treatment difference in the proportion of patients developing abnormal scars in patients having some other lesion surgically treated.

Clinical examples from the trial are shown in Figures 1-6.

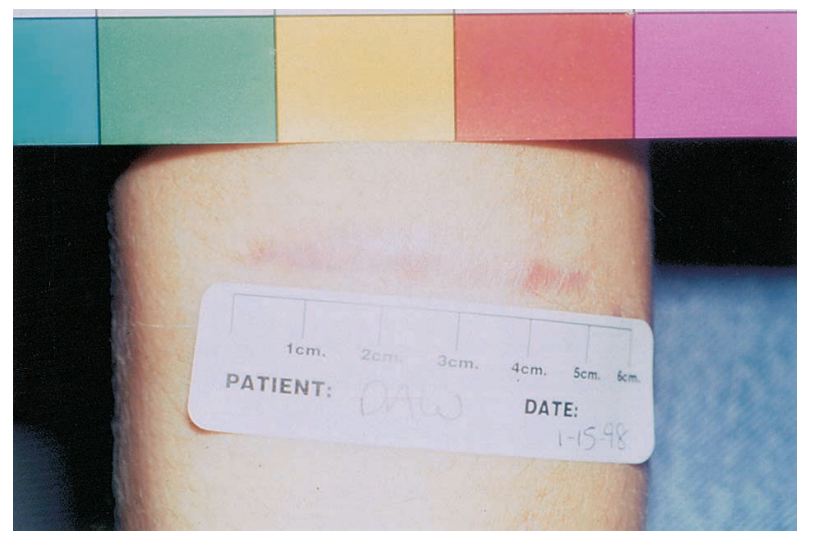

Figure 4. High-risk patient 6 months post-surgery with topical silicone gel sheets. 


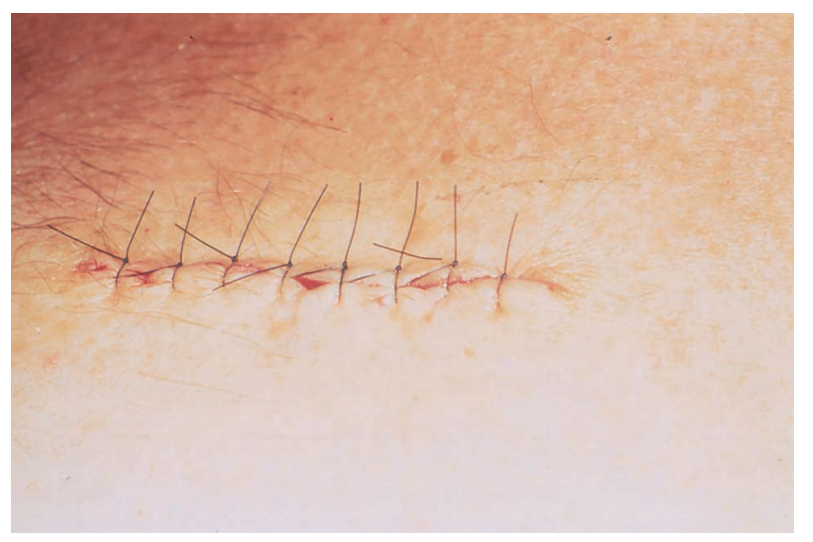

Figure 5. High-risk patient immediately post-surgery.

From this single-center, controlled, randomized clinical trial, it can be successfully argued that the use of topical silicone gel sheets prophylactically following routine dermatologic surgical procedures where sutures are placed may prevent the development of hypertrophic scars and keloids in patients undergoing scar revision. With the number of patients completing the study, it is important for additional work to duplicate the results presented in this manuscript. The small number of patients in each group may effect the interpretation of the data.

As expected, the results from the trial showed no clinical or statistical difference in the formation of abnormal scarring in the low-risk patients, that is, those without any significant history of abnormal scarring. However, in the high-risk group, that is, those patients who had a history of abnormal scarring, topical silicone gel sheets showed some statistical evidence $(\mathrm{P}=.072)$ that using the sheets may lower the incidence of hypertrophic scar or keloid formation in susceptible individuals.

Also of note were differences in those patients having scar revisions and utilizing topical silicone gel

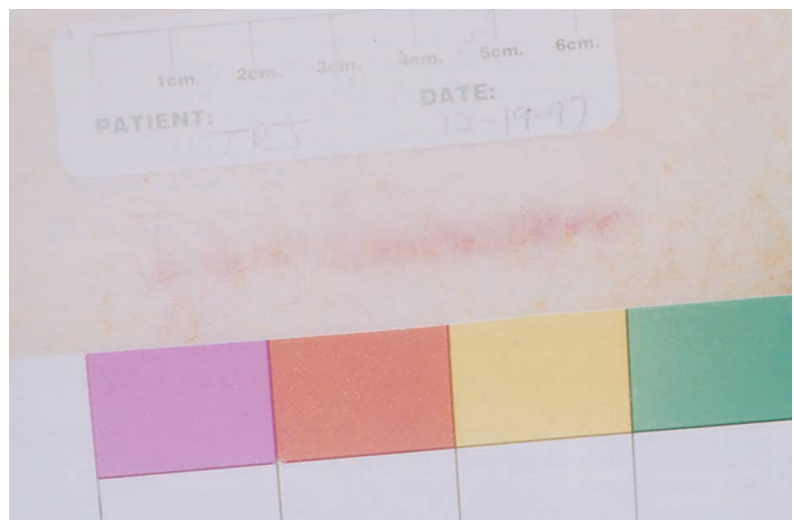

Figure 6 . High-risk patient 6 months post-surgery with topical silicone gel sheets. sheets prophylactically following the procedure. In the group of patients receiving routine postoperative care after scar revision, $83 \%$ (10 patients) developed an abnormal scar versus $36 \%$ (4 patients) who received topical silicone gel sheets. The $P$ value of .35 suggests that topical silicone gel sheets greatly reduce the incidence of recurrences of hypertrophic scars or keloids in this group.

\section{Conclusion}

From the data presented, it appears that topical pure silicone gel sheets are a useful modality in the prevention of hypertrophic scars and keloids in patients undergoing scar revision. The proposed mechanism of action remains through hydration and occlusion of the treated area. Further studies may be necessary to confirm the results presented in this article. However, with an approximate 20 -year track record for efficacy, topical silicone gel sheets are useful adjuncts for dermatologic surgeons-and now should be routinely considered following the scar revision procedure to perhaps prevent abnormal scars from forming, especially in susceptible individuals.

\section{References}

1. Perkins K, Davey RB, Wallis KA. Silicone gel: a new treatment for burn scars and contractures. Burns 1982;9:406-10.

2. Quinn KG, Evans JH, Courtney JM, Gaylor JDS, Reid WH. Nonpressure treatment of hypertrophic scars. Burns 1985;12:102-8.

3. Quinn KG. Silicone gel in scar treatment. Burns 1987;13:S33-S40.

4. Davey RB. The use of silicone gel and Silastic foam in burn scar management-how does it work? Presented at the $7^{\text {th }}$ Congress of the International Society for Burn Injuries, Melbourne, Australia, February 1986.

5. Ahn ST, Monafo WW, Mustoe TA. Topical silicone gel: a new treatment for hypertrophic scars. Surgery 1989;4:781-7.

6. Mercer NSG. Silicone gel treatment of keloid scars. Br J Plast Surg 1989;42:83-7.

7. Gold MH. Topical silicone gel sheeting in the treatment of hypertrophic scars and keloids: a dermatologic experience. J Dermatol Surg Oncol 1993;19:912-6.

8. Katz BE. Silastic gel sheeting is found to be effective in scar therapy. Cosmet Dermatol 1992;5:32-4.

9. Gold MH. A controlled clinical trial of topical silicone gel sheeting in the treatment of hypertrophic scars and keloids. J Am Acad Dermatol 1994;30:506-7.

10. Hirshowitz B, Ullmann Y, Har-Shai Y, Vilenski A, Peled IJ. Silicone occlusive sheeting (SOS) in the management of hypertrophic and keloid scarring, including the possible mode of action, by static electricity. Eur J Plast Surg 1993;16:5-9.

11. Fulton JE. Silicone gel sheeting for the prevention and management of evolving hypertrophic and keloid scars. Dermatol Surg 1995;21: 947-51.

12. Sawada Y, Sone K. Treatment of scars and keloids with a cream containing silicone oil. Br J Plast Surg 1990;43:683-8.

13. Carney SA, Cason CG, Gower JP, et al. Cica-Care gel sheeting in the management of hypertrophic scarring. Burns 1994;20:163-7.

14. Ricketts CH, Martin L, Faria DT, Saed GM, Fwenson OP. Cytokine mRNA changes during the treatment of hypertrophic scars with silicone and nonsilicone gel dressings. Dermatol Surg 1996;22:955-9. 